\title{
Prevalence and type distribution of human papillomavirus in a Chinese urban population between 2014 and 2018: a retrospective study
}

\author{
Mei-Yan Xu ${ }^{1}$, Bing Cao ${ }^{2}$, Yan Chen ${ }^{3}$, Juan Du ${ }^{4}$, Jian Yin ${ }^{1}$, Lan Liu ${ }^{5}$, Qing-Bin Lu ${ }^{\text {Corresp. } 4,6}$ \\ 1 Department of Nutrition, Aerospace Center Hospital, Beijing, Beijing, China \\ 2 Key Laboratory of Cognition and Personality (SWU), Faculty of Psychology, Ministry of Education, Southwest University, Chongqing, Chongqing, China \\ 3 Dalla Lana School of Public Health, University of Toronto, Toronto, ON, Canada \\ 4 Department of Laboratorial Science and Technology, School of Public Health, Peking University, Beijing, Beijing, China \\ 5 Department of Health Management, Aerospace Center Hospital, Beijing, Beijing, China \\ 6 Beijing Key Laboratory of Toxicological Research and Risk Assessment for Food Safety, Beijing, P.R. China, Beijing, Beijing, China \\ Corresponding Author: Qing-Bin Lu \\ Email address: qingbinlu@bjmu.edu.cn
}

Background Human papilloma virus (HPV) infection is the most common sexually transmitted infections among women worldwide. The current study's main objective is to report the prevalence and distribution of HPV types in an urban population in Beijing, China. Methods All the eligible female participants aged $\geq 18$ years were recruited from the Aerospace Center Hospital in Beijing, China between 2014 and 2018. A total of 21 HPV types were detected by the polymerase chain reaction (PCR) reverse dot blot method and fluorescence quantitative PCR method. Results In total, 12 high risk HPV types and nine low risk HPV types were detected. The HPV-positive rates were $8.85 \%$ in $2014,7.16 \%$ in $2015,7.60 \%$ in $2016,8.31 \%$ in 2017 , and $7.72 \%$ in 2018 , respectively, in an urban population in Beijing, China. Overall, no significant differences in the HPV-positive rates were found over the five years. The peak prevalence of HPV infection in all types was observed in age group of 20-24 in all types. HPV52 was the dominant HPV type across the five years. Among all 21 HPV types, HPV66, HPV26, and HPV59 were ranked the top three in coinfection occurrence. Conclusions Our findings are great helpful for HPV screening and vaccination., and the associations between gynaecological diseases and the HPV types with high prevalence, particularly HPV52, warrant further investigation. 
1 Prevalence and type distribution of human papillomavirus in a Chinese urban population

2 between 2014 and 2018: a retrospective study

3

4 Mei-Yan $\mathrm{Xu}^{1, \#}$, Bing $\mathrm{CaO}^{2, \#}$, Yan $\mathrm{Chen}^{3}$, Juan $\mathrm{Du}^{4}$, Jian $\mathrm{Yin}^{1}$, Lan Liu ${ }^{5, *}$, Qing-Bin $\mathrm{Lu}^{4,6^{*}}$

5

$6{ }^{1}$ Department of Nutrition, Aerospace Center Hospital, Beijing, P. R. China

$7 \quad{ }^{2}$ Key Laboratory of Cognition and Personality (SWU), Faculty of Psychology, Ministry of

8 Education, Southwest University, Chongqing, P. R. China

$9{ }^{3}$ Dalla Lana School of Public Health, University of Toronto, Toronto, ON, Canada

$10{ }^{4}$ Department of Laboratorial Science and Technology, School of Public Health, Peking

11 University, Beijing, P. R. China

$12{ }^{5}$ Department of Health Management, Aerospace Center Hospital, Beijing, P. R. China

$13{ }^{6}$ Beijing Key Laboratory of Toxicological Research and Risk Assessment for Food Safety,

14 Beijing, P.R. China

15

16 \#Mei-Yan Xu and Bing Cao contributed equally.

18 Correspondence address

19 Qing-Bin Lu, M.D.,

20 Department of Laboratorial Science and Technology, School of Public Health, Peking

21 University, Beijing, P.R. China.

22 Tel (fax): +86-10-82805327. Email: qingbinlu@bjmu.edu.cn

23 Lan Liu, Professor, 
24 Department of Health Management, Aerospace Center Hospital, Beijing, P.R. China. Tel (fax):

25 +86-10-59971711. Email: 721Lancy@sina.com

26

27 Running title

28 HPV prevalence

29 
30

31

32

33

34

35

36

37

38

39

40

41

42

\section{Abstract}

\section{Background}

Human papilloma virus (HPV) infection is the most common sexually transmitted infections among women worldwide. The current study's main objective is to report the prevalence and distribution of HPV types in an urban population in Beijing, China.

Methods

All the eligible female participants aged $\geq 18$ years were recruited from the Aerospace Center Hospital in Beijing, China between 2014 and 2018. A total of 21 HPV types were detected by the polymerase chain reaction (PCR) reverse dot blot method and fluorescence quantitative PCR method.

\section{Results}

In total, 12 high risk HPV types and nine low risk HPV types were detected. The HPV-positive rates were $8.85 \%$ in $2014,7.16 \%$ in $2015,7.60 \%$ in $2016,8.31 \%$ in 2017 , and $7.72 \%$ in 2018 , respectively, in an urban population in Beijing, China. Overall, no significant differences in the HPV-positive rates were found over the five years. The peak prevalence of HPV infection in all types was observed in age group of 20-24 in all types. HPV52 was the dominant HPV type across the five years. Among all 21 HPV types, HPV66, HPV26, and HPV59 were ranked the top three in coinfection occurrence.

\section{Conclusions}

Our findings are great helpful for HPV screening and vaccination., and the associations between gynaecological diseases and the HPV types with high prevalence, particularly HPV52, warrant further investigation. 


\section{Introduction}

Human papilloma viruses (HPV) are small groups of double-stranded DNA viruses without enveloped icosahedral capsids (Boda et al. 2018; zur Hausen 2002) that cause the most common sexually transmitted infections among women worldwide (Handler et al. 2015; Satterwhite et al. 2013). More than 200 HPV types have been identified (Ghedira et al. 2016) and some had been confirmed to be associated with certain diseases, mainly cancers. Replicated evidence support that HPV infection causes cervical cancer in virtually $100 \%$ of cases and it is associated with the development of an important percentage of penile, vaginal, anal, vulvar, and oropharyngeal cancers and pre-cancers (Bansal et al. 2016). Epidemiological research has reported that 5.2\% of all cancers worldwide can be attributed to HPV infection (Steben \& Duarte-Franco 2007).

Moreover, the overall annual direct medical cost burden of preventing and treating HPVassociated diseases was estimated as an annual $\$ 8.0$ billion in the United States in 2010 (Chesson et al. 2012).

Each of the HPV type is identified with a number corresponding to the sequence of discovery. The International Agency for Research on Cancer (IARC) working group classified the following12 HPV types as carcinogenic to humans (Group 1) and high risk HPV (HR-HPV): including HPV16, HPV18, HPV31, HPV33, HPV35, HPV39, HPV45, HPV51, HPV52, HPV56, HPV58, and HPV59 (Clifford et al. 2011).

HPV type prevalence varies between different countries, but also within different regions of the same country (Wang et al. 2015). A meta-analysis reported that the estimated global HPV prevalence among women of all ages is $11.7 \%$. The areas with the highest prevalence are SubSaharan Africa (24.0\%), Eastern Europe (21.4\%), and Latin America (16.1\%), respectively (Bruni et al. 2010). The prevalence of HPV infections among mainland Chinese women is 
reported as $11.0 \%$ (Zhou et al. 2018), which is similar to the average level worldwide. Additional factors such as age, marital status, and underlying coinfection such as with human immunodeficiency virus (HIV), were reported to be associated with HPV infections (Shi et al. 2016).

HPV prevalence is still a concerning since there is no HPV vaccine that covers all HPV types associated with cervical cancer. Previous epidemiological studies have mainly focused on the prevalence of HR-HPV types with specific cancers, and updated information on type-specific HPV prevalence and distribution in a general population is warranted. Therefore, we conducted a retrospective, hospital-based study to estimate the overall prevalence and distribution of HPV types in Beijing, China. The persistence rates of HPV infection after a 3-year follow up were also determined. The current findings will be valuable in the development and application of HPV vaccines in China.

\section{Methods}

\section{Study design and participants}

For this hospital-based retrospective epidemiological study, participants were recruited from the Aerospace Center Hospital located in Beijing, China between 2014 and 2018. The inclusion criteria of our subjects were as follows: 1) female, age 18 years old or older; 2) participating in annual health examinations and having HPV detected result at least once at the current hospital during our research period. We excluded 1) pregnant and postpartum women; and 2) the people who have been diagnosed with any kind of cancer. For our research samples, some had participated in annual health examinations every year, and some only participated once during our study period. The subject had a healthy examination once a year and had one test for HPV 
99 infection. We included all available data in the statistical analysis. We conducted a chart review

100 to collect the recruited subjects' age.

101 The research protocol was approved by the human ethics committee of the Aerospace Center

102 Hospital and all methods were carried out in accordance with the approved guidelines (No.

103 2014YN-01). All participants provided written or verbal informed consent to have their samples

104 and information collected and used for this study.

105 Outcome measurement

106 Cervicovaginal cellular swabs were collected by a doctor during gynecological healthy

107 examination. Most samples were tested on the same day after collection; otherwise, they were

108 stored at $-70^{\circ} \mathrm{C}$ until tested.

109 Viral DNA was isolated from cervicovaginal cellular swabs using a QIAamp DNA Mini Kit

110 (QIAGEN). The 15 HPV types were detected using the polymerase chain reaction (PCR)

111 reverse dot blot method based on L1 region with the HPV Genotyping Kit [Yaneng

112 Biotechnology (Shenzhen) Co., Ltd, Shenzhen, China], which enabled the detection of 12 HR-

113 HPV types (HPV16, 18, 31, 33, 35, 39, 45, 51, 52, 56, 58 and 59) and three low risk HPV (LR-

114 HPV) types (HPV6, 11 and 68) during 2014-2016. An aliquot of a $5 \mu L$ DNA sample was briefly

115 used, and the PCR reaction was amplified in a thermal cycler under the following conditions: an

116 initial $15 \mathrm{~min}$ at $50^{\circ} \mathrm{C}, 10 \mathrm{~min}$ at $95^{\circ} \mathrm{C} ; 40$ cycles of $30 \mathrm{~s}$ at $94^{\circ} \mathrm{C}, 90 \mathrm{~s}$ at $42^{\circ} \mathrm{C}$, and $30 \mathrm{~s}$ at $72^{\circ} \mathrm{C}$;

117 and a final extension $5 \mathrm{~min}$ at $5 \mathrm{~min}$. The PCR products were immobilized onto a nitrocellulose

118 membrane and hybridized with typing probes. Final results were judged by the direct

119 visualization of the location of blue spots located on the membrane. During 2014-2018, 21 HPV

120 types were detected using the fluorescence quantitative PCR method with a nucleic acid

121 detection kit (Jiangsu Shuoshi Biotechnology Co., Ltd, Taizhou, China) that enables the 
122 detection of 12 HR-HPV types (HPV16, 18, 31, 33, 35, 39, 45, 51, 52, 56, 58 and 59) and nine

123 LR-HPV types based on E region (HPV06, 11, 26, 53, 58, 66, 73, 81 and 82; HPV26, 53, 66, 73

124 and 82 were considered as LR-HPV types in this study). Each PCR reaction was performed

125 briefly in a $40 \mu \mathrm{L}$ mixture containing $4 \mu \mathrm{L}$ of extracted DNA and $36 \mu \mathrm{L}$ of PCR master mix, at

$12694^{\circ} \mathrm{C}$ for $2 \mathrm{~min}, 40$ cycles of $10 \mathrm{~s}$ at $93^{\circ} \mathrm{C}$, and $30 \mathrm{~s}$ at $62^{\circ} \mathrm{C}$. Specific primers and correspondent

127 fluorescent probes were designed to detect 21 HPV types in the assay. A single copy gene was

128 amplified to serve as an internal quality control for DNA preparation. A sample was determined

129 as HPV positive when the cycle threshold ( $\mathrm{t}$ value) was less than or equal to 38.0 , and the

130 amplification curve was a typical S-type. According to the preliminary experiments in the

131 manuals, the sensitivity and specificity in both the two kits were more than $98 \%$

132 (http://www.yanengbio.com/HPV/ and http://www.s-

133 sbio.com/products detail/productId=512.html). Detailed information on the two detection

134 methods is listed in the Supplemental Table 1.

135 Statistical analysis

136 Annual HPV prevalence rates and the distribution of HPV types in each year were calculated

137 using positive rates or proportions and 95\% confidence intervals (CIs), and they were stratified

138 by age at diagnosis. We explored the differences of HPV detection across age using either an

139 independent t-test. A positive HPV sample was defined as having an infection of HPV types

140 equal or more than one. A single infection was defined as being infected by one type of HPV

141 infection. The coinfection proportion was defined as the number of subjects infected with more

142 than one HPV type divided by the total number of subjects infected with at least one HPV type.

143 The persistence rate of HPV infection was calculated as (1) + and (2)+)/(1)+ and (2)+/-) for one

144 year persistence, (1) + and (2) + and (3)+)/(1)+ and (2) $+/-$ and (3)+/-) for two-year persistence and 
145 (1) + and (2) + and (3) + and (4) +$) /((1)+$ and (2) $+/-$ and (3) $+/-$ and (4) $+/-)$ for three-year persistence,

146 where (1), (2), (3), and (4) mean the detection at the first, second, third and fourth year,

147 respectively, and + means HPV positive, and +/- means HPV positive or negative. A two-sided $p$

148 value of less than 0.05 was considered significant. All statistical analyses were done using Stata

14914.0 (Stata Corp LP, College Station, TX, USA).

150

151 Results

152

153

154

155

156

157

158

159

160

161

162

163

164

165

166

167

\section{Overall prevalence and basic characteristic distribution of HPV infection}

Between January 1, 2014 and August 31, 2018, 5880 women involved in this study had at least one HPV detection (Supplemental Fig. 1). The annual age distributions of the participants every year are shown in Supplemental Fig. 2. The age distribution was similar across every year and the majority of participants were between 30-45 years old. As shown in Fig.1, the HPV positive rates were $8.85 \%$ in $2014,7.16 \%$ in $2015,7.60 \%$ in $2016,8.31 \%$ in 2017 , and $7.72 \%$ in 2018, respectively. No significant differences in HPV positive rates were found over the five years $(p=0.357)$. The median age of the participants between HPV $(+)$ and HPV $(-)$ cases are were comparable in the five years, respectively (Table 1).

\section{HPV type distribution}

Twelve HR-HPV and nine LR-HPV types were detected, the HR-HPV rate decreased over time (Fig. 2A). The detection rate of LR-HPV types increased, which may be caused by the dissimilarity of the assays, although the detection rates for HPV06 and HPV11 were comparable between the two HPV detection methods (Supplemental Table 1). The HPV positive rates varied across different age groups. Here we identified the age distribution of HPV infection. As shown in Fig. 2B, most HPV infections occurred in age groups of 20-24 years old, 65-69 years

Peer) reviewing PDF | (2019:09:40939:3:0:NEW 6 Jan 2020) 
168 old, and 50-54 years old. Young women aged 20-24 years had the highest LR-HPV and HR-

169 HPV infection rates (Fig. 2C and 2D), and the detection rates of HPV06 and HPV11 also peaked

170 in the 20-24 years old age group (Fig. 2E).

171 The distributions of positive rate of all 21 detected HPV types were different in each year

172 (Fig. 3 and Supplemental Fig. 3). The most dominant HPV type was HPV52, which occupied

173 the top position all five years. HPV58, HPV16, and HPV53 were also ranked high but their order

174 slightly changed each year.

175 Coinfection and persistence of HPV types infection

176 Multiple HPV infections were very common, and the ranking of HPV type by coinfection

177 status is shown in Fig. 4. About 75\% of the HPV types had a coinfection proportion $\geq 50 \%$.

178 Among all 21 HPV types, HPV66, HPV26, and HPV59 were ranked the highest in coinfections.

179 HPV52 had the most positive rate but was ranked the lowest. The distribution of double HPV

180 coinfections is listed in Supplemental Table 2.

181 Table 2 shows the HPV positive rates in the follow-up periods after the first HPV positive

182 test. Significant differences in HPV positive rates were found in both the persistent infection

183 group and the non-persistent infection group during the 3 -years follow-up ( $<<0.001)$. Persistent

184 infections of different HPV types in four different years were detected in 16 cases. As shown in

185 Supplemental Fig. 4, HPV52, HPV58, HPV35, and HPV68 were more likely to cause persistent 186 infections.

Discussion

The main objective of this retrospective study was to report the prevalence and distribution of

HPV types based on the annual health examinations and HPV detection results of women aged $\geq$ 
19118 years between 2014 and 2018 at the Aerospace Center Hospital in Beijing, China. The second

192 objective of the study was to with more information in order to protect women from further HPV 193 infections and related cancers in China. Most previous studies mainly focused on HR-HPV types 194 (Guardado-Estrada et al. 2014; Nielsen et al. 2009). To the best of our knowledge, this is the first 195 large-scaled study to provide the prevalence, distribution, and persistence of both HR-HPV and 196 LR-HPV types in an urban population in China.

197 The prevalence rate of HPV infection revealed no changes over the years, which means that 198 HPV infection exists steadily and persistently, sustainably damaging women's health. The 199 prevalence rate of HPV infection can be controlled and reduced through the use of HPV 200 vaccines, making the promotion and acceptance of HPV vaccines crucial.

201 The prevalence rate of HR-HPV types shows a slightly decreasing trend overall in all ages 202 over the five years between 2014 and 2018. The detection rate of LR-HPV types, however, 203 shows an overall increasing trend, which may be expected due to the dissimilarity of the assays. 204 Therefore, the variety of HPV infections were increased, although the prevalence rate of HPV 205 infection was reduced. In regard to the distribution of HPV infections across different age 206 groups, the peak prevalence of all HR-HPV and LR-HPV types of HPV infections was observed 207 in the 20-24 years old age group. The prevalence of LR-HPV types in women aged 20-24 years 208 is close to $6 \%$, which is significantly high compared to less than $2 \%$ observed in other age 209 groups. Women aged 65-69 years also showed significantly high prevalence in all HR-HPV 210 types. However, these results may be biased by the different detection methods used in this 211 study.

212 Because of the large population base of China, there is imbalanced development and 213 inconsistent allocations of healthcare resources and public health awareness (Qiao 2018). A 
214 previous study showed that women aged 40-49 years were the peak age group for HPV infection

215 prevalence, accounting for $17.9 \%$ of cases in in Ningbo, south-eastern China (Baloch et al.

216 2017). According to a study performed in Yunnan, south-western China, women with the highest

217 HPV prevalence were $\leq 29$ years old in the urban regions and $\geq 50$ years in rural regions. These

218 studies did not achieve uniform results on age distribution (Baloch et al. 2017). In addition to

219 age, location (i.e. rural/ urban areas) and healthcare resources, other risk factors may contribute

220 to the prevalence and distribution of HPV, such as marital status, sexual activity, genetic

221 variants, and coinfections with other diseases (i.e. HIV) (Zhao et al. 2018), and education, public

222 health awareness, alcohol use, and tobacco use may be potential confounding variables (Baloch

223 et al. 2017).

224 Over the five-year period, HPV52 was the most dominant type among patients with positive

225 detections, supporting the findings of several other studies (Baloch et al. 2017; Hong et al. 2015).

226 It is followed by HPV58 and HPV16 as the most common types in female residents in Shanghai

227 (Xu et al. 2018). In addition to single infections caused by HPV types, HPV52 also accounted for

228 over $80 \%$ of coinfection cases, followed by HPV58 and HPV16. HPV26, HPV73 and HPV82

229 had the lowest single infection/coinfection proportions. The high prevalence of HPV52 in our

230 study population and its association with other gynecological diseases warrant further

231 investigation.

232 According to the previously reported data, over $70 \%$ of women have had an HPV infection at

233 least once in their lifetime, with about 10\% having a lifelong persistent infection (Liao et al.

234 2018). Persistent HPV infection can cause abnormal proliferation of cells, and the accumulations

235 of genetic damage leading to cancer of the cervix, vulva, vagina, anus, penis, and/or oropharynx

236 (Crosbie et al. 2013; Hong et al. 2015). It is the second most common cause of death from cancer 
237 for women globally (Lowy \& Schiller 2006), with higher death rates reported in lower income

238 countries (Qiao 2018). According to our findings, persistent infection contributed to the positive

239 rate of HPV detection over a three-year follow-up. High-risk HPV16 and HPV18 account for

240 over $70 \%$ of cases of cervical cancer, which is the fourth most common cancer among women

241 worldwide (Qiao 2018), while LR-HPV06 and LR-HPV11 account for about 90\% of external

242 genital warts cases (Lowy \& Schiller 2006).

243 Prevention methods such as screening, early-detection and treatment, and vaccination are

244 used to fight HPV viruses(Qiao 2018). Three types of vaccinations are available in mainland

245 China: the bivalent vaccine which targets HPV16 and HPV18; the tetravalent vaccine which

246 targets HPV6, HPV11, HPV16 and HPV18; and the nine-valent (9v) vaccine targets five

247 additional HR-HPV types (HPV31, HPV33, HPV45, HPV52 and HPV58) (Crosbie et al. 2013;

248 Lowy \& Schiller 2006). The 9v vaccine (Gardasil 9) was approved by the Food and Drug

249 Administration of China and was recently released into the market on April. 28, 2018. A set of

250 three vaccines costs about 5,800 CNY, which is about twice the price in North America countries

251 (i.e. America, Canada). Due to limited quantities imported from the United States to a high

252 number of clinics and hospitals, vaccines are scare in China. Women 25 years and older may be

253 able to afford the cost and long wait time, but younger women may not be able to afford the

254 vaccines themselves if their healthcare programs do not cover the cost. Implementing HPV

255 prevention initiatives may not meet the current need (Ogembo et al. 2015).

256 All data in the current study are based on highly reliable hospital records. The large sample size-

257 based population means the results of this study are generalizable. Limited data are available on

258 the prevalence and type distribution of HPV infections in Beijing, the capital of China making

259 the present study valuable for the future clinical researches of HPV screening and anti-HPV 
260 vaccines. HPV distribution largely varies and is affected by many confounding variables. The

261 results and interpretations presented in the current study should be considered in the context of

262 the following limitations. First, all participants were recruited from the Aerospace Center

263 Hospital in Beijing and most of them were from urban areas, which is not sufficiently

264 representative of the whole population and may be selection biased. Second, since individuals in

265 China can choose their type of physical examination from a variety of packages and can

266 voluntarily choose or decline HPV type testing, a large number of people do not have the results

267 from all five years. Additionally, we can only perform analysis of persistent infection in a small

268 number of participants with follow-up test results. A detection bias for some HPV types exists in

269 the study due to changing and unbalanced distributions of detection methods, especially for the

270 LR-HPV types, which may be related to changes in LR- or HR-HPV types.

271

272 Conclusion

273 The current retrospective study presents the prevalence and distribution of HPV types in women

274 between 2014 and 2018 in an urban population of Beijing, China. HPV52 was the most dominant

275 HPV type over all five years. The peak prevalence of all HPV infection types was observed in

276 ages 20-24 years old in all types. The current findings can be significant for the future of HPV

277 screening and vaccination. The associations between gynaecological diseases and HPV types

278 with high prevalence, especially HPV52, warrant further investigation. 
280

281

282

283

284

285

286

287

288

289

290

291

292

293

294

295

296

297

298

299

300

301

302

\section{Declarations}

\section{Ethics approval and consent to participate}

The research protocol was approved by the human ethics committee of the Aerospace Center Hospital and all methods were carried out in accordance with the approved guidelines (No.

2014YN-01). All participants provided written or verbal informed consent to have their samples and information collected and used for this study.

\section{Competing interests}

The authors declare that they have no competing interests.

\section{Funding}

The study was supported by National Natural Science Foundation of China (No. 81703274), China Mega-Project for Infectious Diseases Grant (2017ZX10103004), the Youth Innovation Funding (2014QN09) and Peking University Medicine Seed Fund for Interdisciplinary Research (BMU2018MX009). The funders had no role in study design, data collection and analysis, decision to publish, or preparation of the manuscript.

\section{Authors' contributions}

Conceived and designed the experiments: QB Lu, MY Xu, L Liu and B Cao. Collect data and performed the experiments: MY Xu, J Yin and L Liu. Analyzed the data: QB Lu, MY Xu and B Cao. Contributed reagents/materials/analysis tools: QB Lu, J Du, S Wang, Y Chen, MY $\mathrm{Xu}$ and L Liu. Wrote the paper: QB Lu, MY Xu, B Cao, Y Chen and L Liu. 
303 Acknowledgements

$304 \quad$ None.

305 
306

307

308

309

310

311

312

313

314

315

316

317

318

319

320

321

322

323

324

325

326

327

328

329

330

331

332

333

334

335

336

337

338

339

340

341

342

343

344

345

346

347

348

349

350

351

352

\section{References}

Baloch Z, Yasmeen N, Li Y, Ma K, Wu X, Yang SH, and Xia X. 2017. Prevalence and risk factors for human papillomavirus infection among Chinese ethnic women in southern of Yunnan, China. Brazlilian Journal of Infectious Diseases 21:325-332. 10.1016/j.bjid.2017.01.009

Bansal A, Singh MP, and Rai B. 2016. Human papillomavirus-associated cancers: A growing global problem. International Journal of Applied and Basic Medical Research 6:84-89. 10.4103/2229516X.179027

Boda D, Docea AO, Calina D, Ilie MA, Caruntu C, Zurac S, Neagu M, Constantin C, Branisteanu DE, Voiculescu V, Mamoulakis C, Tzanakakis G, Spandidos DA, Drakoulis N, and Tsatsakis AM. 2018. Human papilloma virus: Apprehending the link with carcinogenesis and unveiling new research avenues (Review). International Journal of Oncology 52:637-655. 10.3892/ijo.2018.4256

Bruni L, Diaz M, Castellsague X, Ferrer E, Bosch FX, and de Sanjose S. 2010. Cervical human papillomavirus prevalence in 5 continents: meta-analysis of 1 million women with normal cytological findings. The Journal of Infectious Diseases 202:1789-1799. 10.1086/657321

Chesson HW, Ekwueme DU, Saraiya M, Watson M, Lowy DR, and Markowitz LE. 2012. Estimates of the annual direct medical costs of the prevention and treatment of disease associated with human papillomavirus in the United States. Vaccine 30:6016-6019. 10.1016/j.vaccine.2012.07.056

Clifford GM, Howell-Jones R, and Franceschi S. 2011. Judging the carcinogenicity of human papillomavirus types by single/multiple infection ratio in cervical cancer. International Journal of Cancer 129:1792-1794. 10.1002/ijc.25833

Crosbie EJ, Einstein MH, Franceschi S, and Kitchener HC. 2013. Human papillomavirus and cervical cancer. The Lancet 382:889-899. 10.1016/S0140-6736(13)60022-7

Ghedira R, Mahfoudh W, Hadhri S, Gabbouj S, Bouanene I, Khairi H, Chaieb A, Khelifa R, Bouaouina N, Remadi S, Elmi AA, Bansal D, Sultan AA, Faleh R, Zakhama A, Chouchane L, and Hassen E. 2016. Human papillomavirus genotypes and HPV-16 variants distribution among Tunisian women with normal cytology and squamous intraepithelial lesions. Infectious Agents and Cancer 11:61. 10.1186/s13027-016-0109-2

Guardado-Estrada M, Juarez-Torres E, Roman-Bassaure E, Medina-Martinez I, Alfaro A, Benuto RE, Dean M, Villegas-Sepulveda N, and Berumen J. 2014. The distribution of high-risk human papillomaviruses is different in young and old patients with cervical cancer. PLoS One 9:e109406. 10.1371/journal.pone.0109406

Handler MZ, Handler NS, Majewski S, and Schwartz RA. 2015. Human papillomavirus vaccine trials and tribulations: Clinical perspectives. Journal of the American Academy of Dermatology 73:743-756; quiz 757-748. 10.1016/j.jaad.2015.05.040

Hong H, He TF, Ni HX, Zhang S, and Xu GZ. 2015. Prevalence and genotype distribution of HPV infection among women in Ningbo, China. International Journal of Gynecology \& Obstetrics 131:96-99. 10.1016/j.ijgo.2015.04.027

Liao L, Cheng H, Zeng F, Zhou W, and Ding Y. 2018. Prevalence and distribution of human papillomavirus genotypes among women with high-grade squamous intraepithelial lesion and invasive cervical cancer in Ganzhou, China. Journal of Clinical Laboratory Analysis:e22708. 10.1002/jcla.22708

Lowy DR, and Schiller JT. 2006. Prophylactic human papillomavirus vaccines. Journal of Clinical Investigation 116:1167-1173. 10.1172/JCl28607

Nielsen A, Iftner T, Munk C, and Kjaer SK. 2009. Acquisition of high-risk human papillomavirus infection in a population-based cohort of Danish women. Sexually Transmitted Infections 36:609-615. 10.1097/OLQ.0b013e3181a96d0e

Ogembo RK, Gona PN, Seymour AJ, Park HS, Bain PA, Maranda L, and Ogembo JG. 2015. Prevalence of human papillomavirus genotypes among African women with normal cervical cytology and

Peer) reviewing PDF | (2019:09:40939:3:0:NEW 6 Jan 2020) 
neoplasia: a systematic review and meta-analysis. PLoS One 10:e0122488. 10.1371/journal.pone.0122488

Qiao YL. 2018. A long journey of cervical cancer prevention in China. Chinese Journal of Oncology 40:721-723. 10.3760/cma.j.issn.0253-3766.2018.10.001

Satterwhite CL, Torrone E, Meites E, Dunne EF, Mahajan R, Ocfemia MC, Su J, Xu F, and Weinstock H. 2013. Sexually transmitted infections among US women and men: prevalence and incidence estimates, 2008. Sexually Transmitted Infections 40:187-193. 10.1097/OLQ.0b013e318286bb53

Shi Y, Peng SL, Yang LF, Chen X, Tao YG, and Cao Y. 2016. Co-infection of Epstein-Barr virus and human papillomavirus in human tumorigenesis. Chinese Journal of Cancer 35:16. 10.1186/s40880-0160079-1

Steben M, and Duarte-Franco E. 2007. Human papillomavirus infection: epidemiology and pathophysiology. Gynecologic Oncology 107:S2-5. 10.1016/j.ygyno.2007.07.067

Wang R, Guo XL, Wisman GB, Schuuring E, Wang WF, Zeng ZY, Zhu H, and Wu SW. 2015. Nationwide prevalence of human papillomavirus infection and viral genotype distribution in 37 cities in China. BMC Infectious Diseases 15:257. 10.1186/s12879-015-0998-5

Xu J, Xia Z, Wang L, Yang B, Zhu Y, Zhu X, and Xu L. 2018. Human papillomaviruses 16 and 58 are distributed widely among women living in Shanghai, China, with high-grade, squamous intraepithelial lesions. Epidemiology and Infection:1-6. 10.1017/S0950268818003011

Zhao P, Liu S, Zhong Z, Hou J, Lin L, Weng R, Su L, Lei N, Hou T, and Yang H. 2018. Prevalence and genotype distribution of human papillomavirus infection among women in northeastern Guangdong Province of China. BMC Infectious Diseases 18:204. 10.1186/s12879-018-3105-x

Zhou HL, Zhang W, Zhang CJ, Wang SM, Duan YC, Wang JX, Yang H, and Wang XY. 2018. Prevalence and distribution of human papillomavirus genotypes in Chinese women between 1991 and 2016: A systematic review. Journal of Infection 76:522-528. 10.1016/j.jinf.2018.02.008

zur Hausen H. 2002. Papillomaviruses and cancer: from basic studies to clinical application. Nature Reviews Cancer 2:342-350. 10.1038/nrc798 


\section{Figure legends}

382 Fig. 1 The prevalence rate of HPV from 2014 to 2018.

383 Fig. 2 The prevalence rate of low-risk and high-risk HPV types in different age groups. (A)

384 Prevalence rate of low-risk and high-risk HPV types in each year; (B) prevalence of all HPV

385 types of different age groups; (C) prevalence of low-risk HPV types of different age groups; (D)

386 prevalence of high-risk HPV types of different age groups; (E) prevalence of HPV06 and

387 HPV11.

388 Fig. 3 The annual distributions of every HPV types from 2014 to 2018.

389 Fig. 4 Multiple HPV infections and the ranking of coinfection status of each HPV type.

390 Supplemental Fig. 1 The diagram flowchart of the participants in this study.

391 Supplemental Fig. 2 The number of participants in each age groups during 2014-2018.

392 Supplemental Fig. 3 The annual distributions of every HPV types in each year from 2014 to 3932018.

394 Supplemental Fig. 4 The persistent infections of different HPV types in four different years 395 detected in 16 participants. 
Figure 1

The prevalence rate of HPV from 2014 to 2018

The prevalence rate of HPV from 2014 to 2018

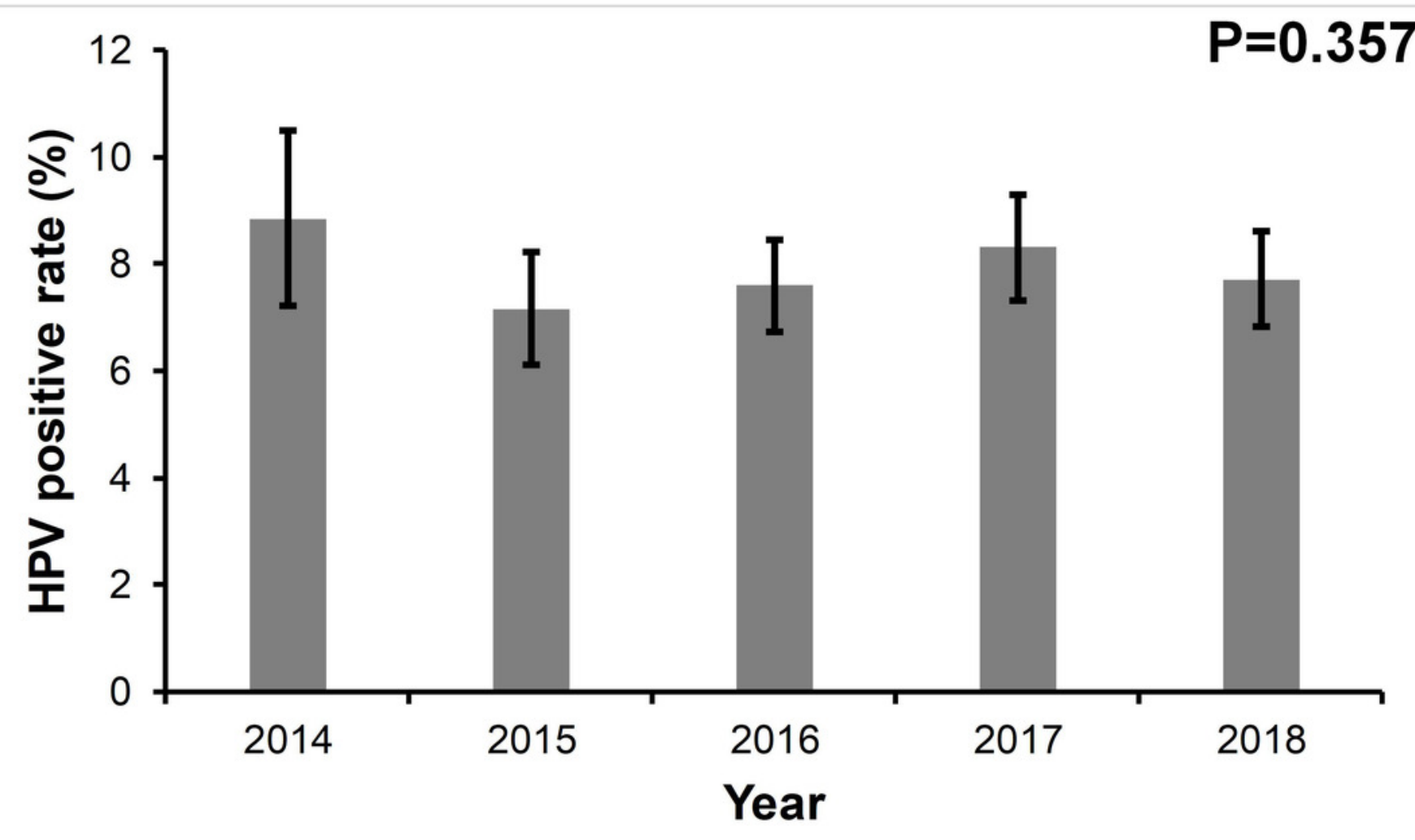


Figure 2

The prevalence rate of low risk and high risk HPV genotypes in different age groups.

(A) prevalence rate of low risk and high risk HPV genotypes in each year; (B) prevalence of all HPV genotypes of different age groups; (C) prevalence of low risk HPV genotypes of different age groups; (D) prevalence of high risk HPV genotypes of different age groups. 

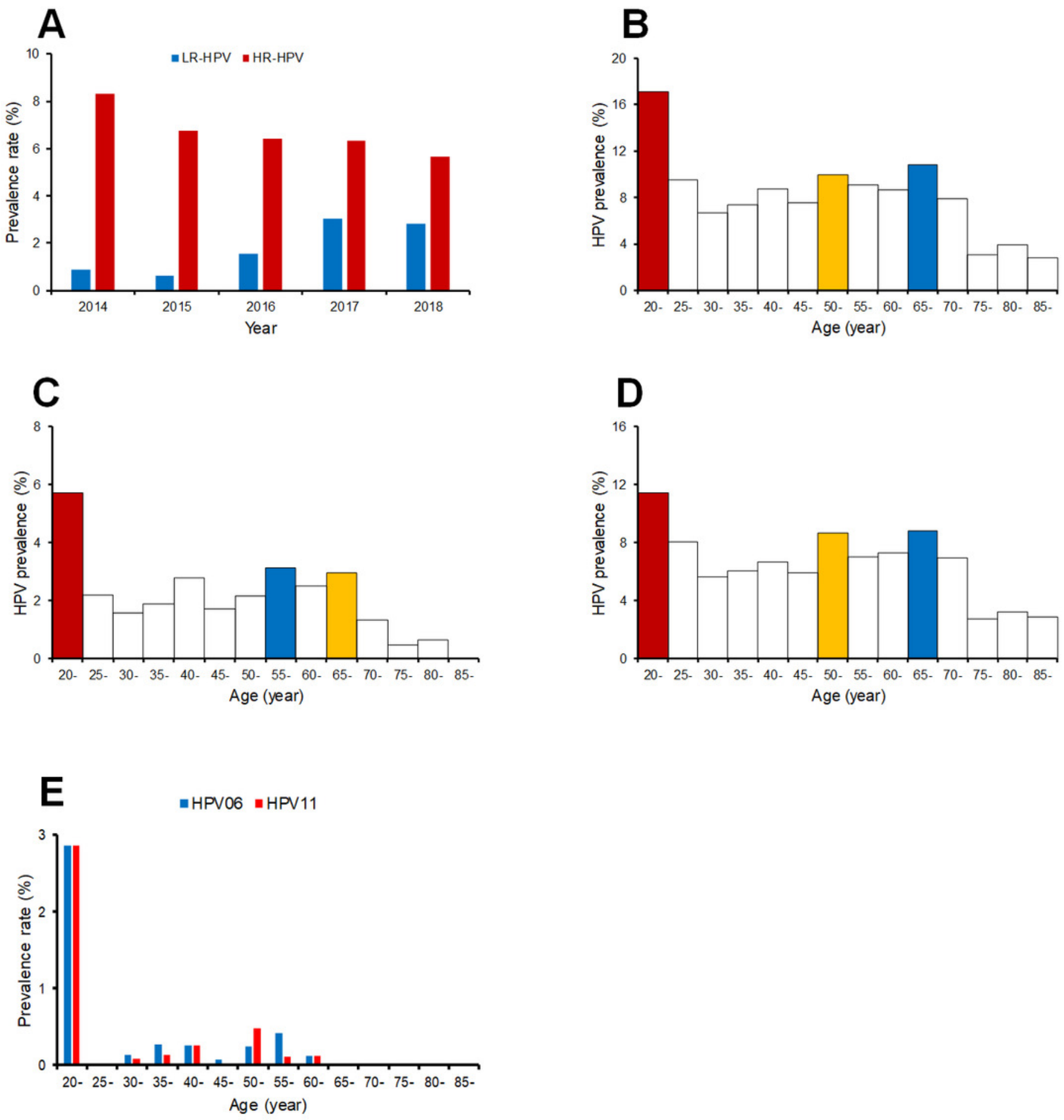
Figure 3

The distributions of every HPV genotypes in each year from 2014 to 2018.

The distributions of every HPV genotypes in each year from 2014 to 2018.

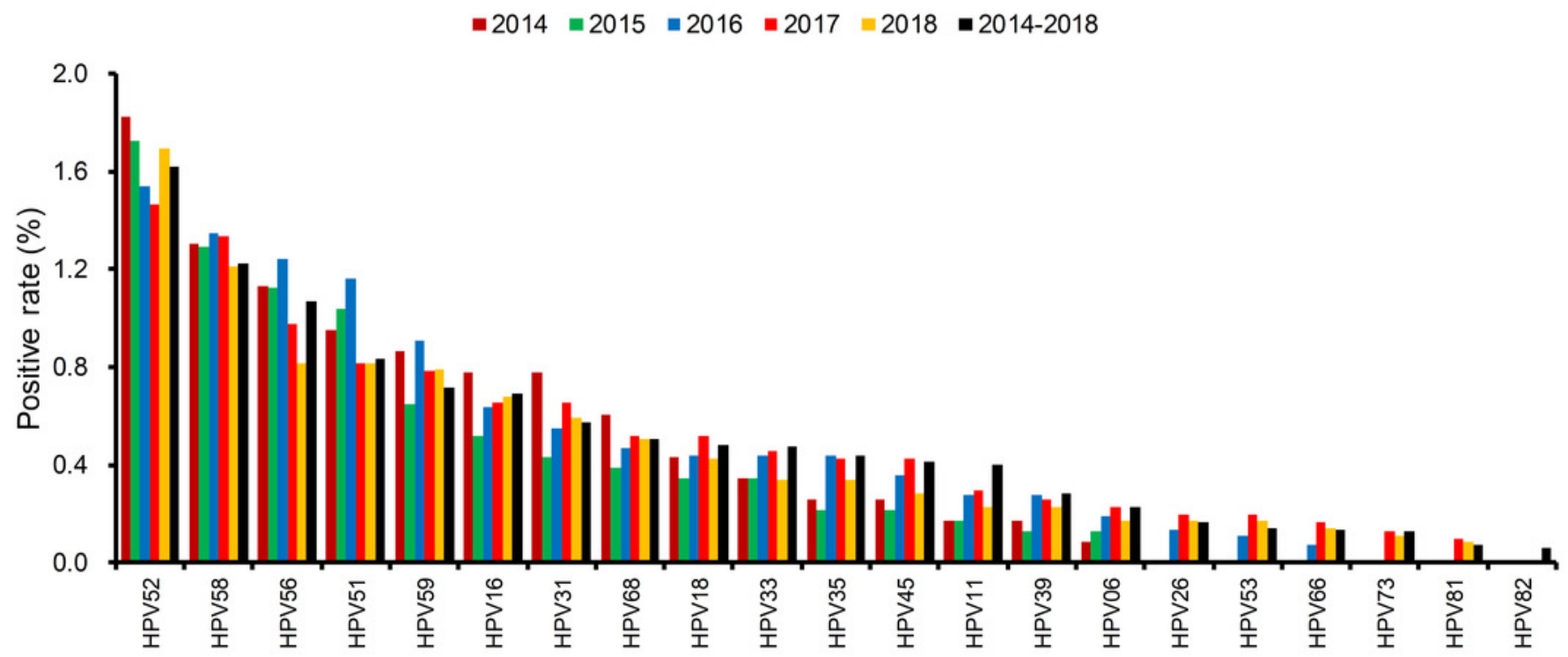


Figure 4

Multiple HPV infections and the ranking of coinfection status of each HPV genotype.

Multiple HPV infections and the ranking of coinfection status of each HPV genotype.

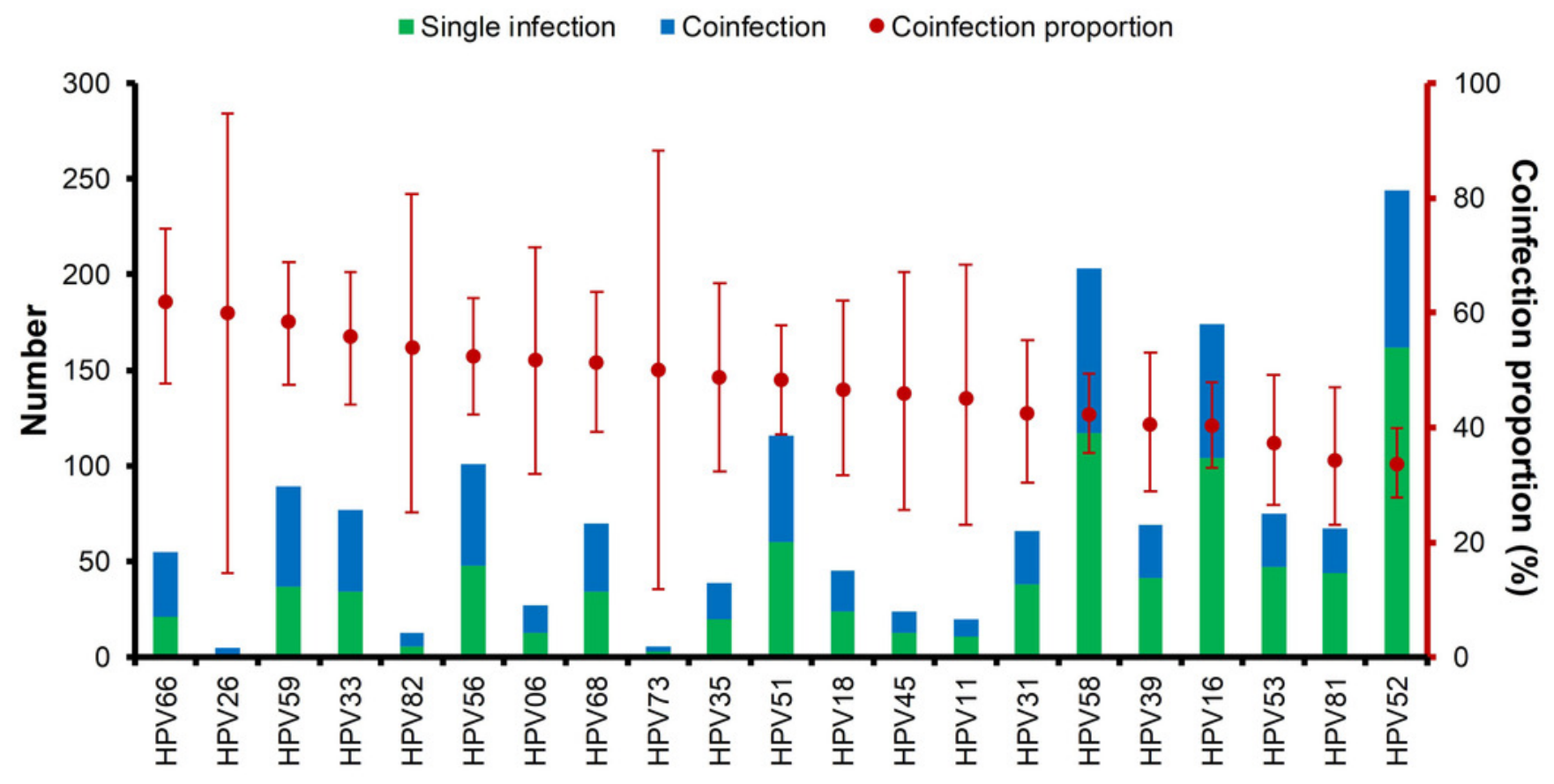




\section{Table $\mathbf{1}$ (on next page)}

Table 1. The basic information of the subjects recruited at the different years in the study

Table 1. The basic information of the subjects recruited at the different years in the study 
1 Table 1. The basic information of the participants recruited at the different years in the study

\begin{tabular}{lcccc}
\hline Variable & Total & HPV $(+)$ & HPV (-) & $P$ \\
\hline Age, years, median (interquartile range) & & \\
2014 & $43(35-56)$ & $46(36-55)$ & $43(35-56)$ & 0.380 \\
2015 & $50(38-64)$ & $52(39-63)$ & $50(38-64)$ & 0.869 \\
2016 & $45(36-58)$ & $45(36-56)$ & $45(36-58)$ & 0.351 \\
2017 & $46(36-60)$ & $45(35-56)$ & $46(36-60)$ & 0.092 \\
2018 & $44(36-57)$ & $45(37-58)$ & $43(36-57)$ & 0.330 \\
\hline
\end{tabular}




\section{Table 2(on next page)}

Table 2. The HPV positive rates in the follow-up for different periods from the first HPV positive

Table 2. The HPV positive rates in the follow-up for different periods from the first HPV positive 
Table 2. The HPV positive rates in the follow-up for different periods from the first HPV positive

\begin{tabular}{ccccc}
\hline Persistent period & Positive number & Total number & Positive rate $(95 \% \mathrm{CI})$ & $\mathrm{P}$ \\
& & & & \\
\hline One year & 186 & 218 & $85.3(79.9-89.7)$ & $<0.001$ \\
Two years & 73 & 118 & $61.9(52.5-70.6)$ & \\
Three years & 73 & 118 & $61.9(52.5-70.6)$ &
\end{tabular}

3 (3) $+/((1)+$ and (2) $+/-$ and (3) $+/-)$ for two-year persistence and (1) + and (2) + and (3) + and (4) +$) /(1)+$ and (2) $+/-$ and (3) $+/-$ and (4) $+/-)$ for three-year

4 persistence, where (1), (2), (3), and (4) mean the detection at the first, second, third and fourth year, respectively, and + means HPV positive, and +/-

5 means HPV positive or negative. 